\title{
LA CRISI DEL TERZO SECOLO E L'EVOLUZIONE DELLE ARTIGLIERIE ROMANE
}

\author{
Salvatore Martino
}

I Romani posero sempre grande attenzione alle macchine da lancio. Le artiglierie delle legioni al tempo di Augusto erano composte da lanciagiavellotti e lanciapietre con la griglia propulsiva realizzata tramite diverse tavole e listelli di legno giuntati insieme secondo uno schema abbastanza complicato, ${ }^{1}$ in linea con la tradizione della poliorcetica ellenistica ma con alcune modifiche (bracci curvi anziché diritti per una maggiore corsa angolare, un minor numero di pezzi per formare la griglia, un profilo ad "ala di gabbiano" in visione dall'alto per la griglia della ballista lanciapietre onde accentuarne la forma palintona ecc.). ${ }^{2}$ Ma già nel primo secolo d.C. un trattatello in greco attribuito ad Erone di Alessandria descrive una lanciagiavellotti di concezione nuova, convenzionalmente chiamata oggi cheiroballistra, la cui griglia propulsiva era realizzata da quattro semplici parti metalliche (due $\kappa \alpha \mu \beta \varepsilon ́ \sigma \tau \rho ı)$ per ospitare i fasci di nervi da porre in torsione, una traversa superiore

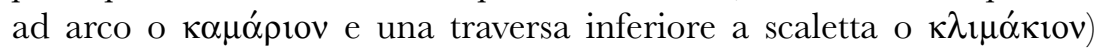
unite in maniera semplice e funzionale. ${ }^{3}$ La vecchia lanciagiavellotti descritta da Vitruvio restò in servizio almeno fino al 69 d.C., ${ }^{4}$ come dimostrano alcuni reperti provenienti da Cremona e ascrivibili alle

${ }^{1}$ Per esse E.W. Marsden, Greek and Roman Artillery: Historical Development (Oxford 1969), 174-187.

2 E. Schramm, Die antiken Geschütze der Saalburg (Berlin 1918), 40-46; Marsden 1969, op. cit. (n. 1), 199-206; E.W. Marsden, Greek and Roman Artillery: Technical Treatises (Oxford 1971), 185-205; L. Callebat et P. Fleury, Vitruve, de l'architecture livre X (Paris 1986), 199-239; A. Wilkins, 'Scorpio and Cheiroballistra', Fournal of Roman Military Equipment Studies 11 (2000), 77-101; F. Russo, Tormenta. Venti secoli di artiglierie meccaniche (Roma 2002), 227-232.

${ }^{3}$ Marsden 1971, op. cit. (n. 2), 206-233; A. Wilkins, 'Reconstructing the Cheiroballistra', Journal of Roman Military Equipment Studies 6 (1995), 5-60; R. Harpham and D.W.W. Stevenson, 'Heron's Cheiroballistra (a Roman Torsion Crossbow)', Journal of the Society of Archer-Antiquaries 40 (1997), 13-17; A. Iriarte, 'Pseudo-Heron's Cheiroballistra: a(nother) reconstruction', Fournal of Roman Military Equipment Studies 11 (2000), 47-75; Russo 2002, op. cit. (n. 2), 232-243.

${ }^{4}$ Vitruvio 10.10.1-6. 
battaglie combattute durante le guerre civili di quell'anno. Dalle campagne di questa città proviene infatti la scudatura frontale bronzea di una lanciagiavellotti Vitruviana, con una iscrizione che ci informa che il pezzo apparteneva alla Legio IIII Macedonica e che fu costruito nel consolato di Marco Vinicio e Tauro Statilio Corvino, cioè il 45 d.C. ${ }^{5}$ La cheiroballistra era, in ogni caso, il modello standard di lanciagiavellotti in servizio nelle legioni almeno dal tempo delle campagne daciche di Traiano, come ci mostra la Colonna traiana, ${ }^{6}$ e lo restò almeno fino ai tempi di Giustiniano. ${ }^{7}$ La sua facilità di assemblaggio, la sua robustezza, la praticità di trasporto, la leggerezza e, non ultima, la potenza (tutte qualità dimostrate dalle ricostruzioni moderne) la rendevano difficilmente migliorabile. ${ }^{8}$ Ampi margini di miglioramento restavano invece per incrementare le prestazioni dei lanciapietre e un paio di reperti archeologici sembrano indicare che proprio il terzo secolo d.C. fu un epoca di sperimentazione in questo senso, che condusse poi ad una soluzione del problema nuova, distaccantesi da tutta la tradizione costruttiva dei pezzi di artiglieria classica.

Nel 1971 vennero ritrovati, fra le rovine dell'antica città di Hatra, nell'attuale Iraq, dei resti metallici di una macchina da lancio. ${ }^{9} \mathrm{I}$ reperti, risalenti al primo quarto del terzo secolo d.C., erano composti da tre flangie, quattro sbarrette di torsione, sei gorbie per le assi della griglia e l'intera scudatura anteriore di protezione e rinforzo, della larghezza di due metri circa. L'aspetto più interessante è che su questa scudatura sono sagomate le due scanalature per alloggiare $\mathrm{i}$ bracci in posizione

${ }^{5}$ D. Baatz, 'Ein Katapult der legio IV Macedonica aus Cremona', Mitteilungen des Deutschen Archäologischen Instituts. Römische Abteilung 87 (1980), 283-299.

${ }^{6}$ Marsden 1969, op. cit. (n. 1), 188-190; Marsden 1971, op. cit. (n. 2), 209.

7 Procopio, De bello Gothico 1.21.14-18; cfr. Marsden 1971, op. cit. (n. 2), 246-248. Ma il modello potrebbe essere restato in servizio nell'esercito bizantino fino a Costantino Porfirogenito ed oltre, dal momento che costui nomina (De administrando Imperio 53.133)

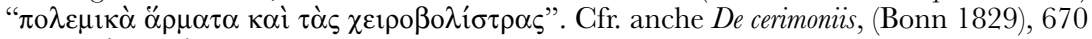

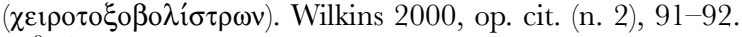

${ }^{8}$ Oltre a Wilkins 1995, op. cit. (n. 3) e Iriarte 2000, op. cit. (n. 3) si vedano anche J. Anstee, 'Tour de Force. An experimental catapult/ballista', Studia Danubiana Simposia 1 (1998), 133-139; A. Zimmermann, 'Zwei ähnlich dimensionierte Torsiongeschütze mit unterschiedlichen Konstruktionsprinzipien: Rekonstruktionen nach Originalteilen aus Cremona (Italien) und Lyon (Frankreich)', Fournal of Roman Military Equipment Studies 10 (1999), 137-140. Interessante è W. Gurstelle, The Art of the Catapult: build Greek Ballistae, Roman Onagers, English Trebuchets and more Ancient Artillery (Chicago 2004).

${ }^{9}$ D. Baatz, 'The Hatra Ballista', Sumer 33 (1977), 141-151; D. Baatz, 'Recent finds of ancient artillery', Britannia 9 (1978), 224-245; D.B. Campbell, 'Auxiliary Artillery Revisited', Bonner Fahrbücher 186 (1986), 117-132. 
di riposo (cioè quando la macchina non è carica). Fin qui nulla di strano, dal momento che le stesse scanalature sono presenti anche sui resti delle piastre di Ampurias e Caminreal: ${ }^{10}$ la cosa insolita è che, mentre nei reperti appena menzionati le scanalature sono laterali (il che è normale, dal momento che laterali erano anche i bracci), nella scudatura di Hatra queste sono sulla parte interna del frontale. Ciò significa che i bracci di questa macchina puntavano in avanti quando essa era scarica e che giravano con la punta verso il fusto e il mirino quando si caricava il pezzo.

Questa eccezionale scoperta ha ridato credito ad una vecchia teoria della seconda metà del diciannovesimo secolo, ${ }^{11}$ secondo la quale le antiche catapulte avevano i bracci mobili verso l'interno. Le rappresentazioni di macchine da lancio che ci sono pervenute dalla civiltà classica (all'epoca non note o non comprese), tutte con bracci verso l'esterno (ad esempio l'altare di Pergamo, ${ }^{12}$ la lapide di Moderato, ${ }^{13}$ ecc.), avevano completamente screditato questa ipotesi: la macchina di Hatra ripropone la questione, nel senso che è possibile che almeno alcune catapulte possono aver avuto bracci mobili verso l'interno.

Il problema è particolarmente sentito per quel che riguarda la cheiroballistra, poiché le uniche antiche rappresentazioni in nostro possesso (quelle sulla Colonna traiana) non mostrano i bracci. Una serie di scoperte archeologiche ci ha fornito diversi reperti di questo pezzo, particolarmente le semi-griglie per i fasci e le traversa superiori ad arco da Orşova, Gornea e Lione, ${ }^{14}$ ma ciò non aiuta a risolvere i problemi perché le semi-griglie (che hanno, lo ricordo, una scanalatura per alloggiare il braccio su una delle barre) sono speculari e possono essere montate indifferentemente a destra o a sinistra della traversa ad

\footnotetext{
${ }^{10}$ Ampurias: Schramm 1918, op. cit. (n. 2), 40-46; Caminreal: J.D. Vicente, M. Pilar Punter y B. Ezquerra, 'La catapulta tardo-republicana y otro equipamiento militar de La Caridad (Caminreal, Teruel)', Journal of Roman Military Equipment Studies 8 (1997), 167-199.

${ }^{11}$ V. Prou, 'La chirobaliste d'Héron d'Alexandrie', Notices et extracts des manuscrits de la Bibliothèque Nationale et autres Bibliothèques 26 (1877), 1-319.

${ }^{12}$ Schramm 1918, op. cit. (n. 2), 35; Marsden 1969, op. cit. (n. 1), tav. 3; D. Baatz, 'Hellenistische Katapulte aus Ephyra (Epirus)', Mitteilungen des Deutschen Archäologischen Instituts. Athenische Abteilung 97 (1982), 211-233.

${ }_{13}$ Marsden 1969, op. cit. (n. 1), 185 e tav. 1; cfr. anche Baatz 1980, op. cit. (n. 5).

${ }^{14}$ Orşova, Gornea: D. Baatz und N. Gudea, 'Teile spätrömischer Ballisten aus Gornea und Orşova (Rumänien)', Saalburg-Fahrbuch 31 (1974), 50-72. Si veda anche Baatz 1978, op. cit. (n. 9), 232-238; Lione: D. Baatz et M. Feugère, 'Éléments d'une catapulte romaine trouvée à Lyon', Gallia 39 (1981), 201-209.
} 
arco. ${ }^{15}$ I reperti di Lione sono perfettamente in linea con la ricostruzione tradizionale; quelli di Gornea sono molto piccoli (le semi-griglie sono alte appena una ventina di centimetri) e forse appartengono ad una manuballista propriamente detta, un pezzo brandito a mano che Vegezio dice armare i tragularii, ${ }^{16}$ fanti leggeri del tardo impero; ${ }^{17} \mathrm{i}$ ritrovamenti più problematici sono quelli di Orşova, probabilmente risalenti alla metà del terzo secolo d.C.

Da questa località rumena provengono, insieme con altri reperti, due semi-griglie alte $36 \mathrm{~cm}$ e larghe $17,50 \mathrm{~cm}$ e una traversa ad arco lunga ben 1,25 m. L'eccessiva lunghezza della traversa si potrebbe spiegare col fatto che i ritrovamenti appartengono a due macchine diverse: ma i sostegni ad "Y" della traversa ad arco si incastrano perfettamente nelle semi-griglie, e ciò lascia poco spazio al dubbio che i pezzi sono della stessa cheiroballistra. Il diametro e la lunghezza delle semi-griglie sono troppo piccoli e la conseguenza è che, con una ricostruzione tradizionale (ovvero con i bracci esterni), il proietto sarebbe stato troppo debole a causa dell'eccessiva distanza dei bracci: pertanto, è stato proposto che essi curvassero verso l'interno e che la lunghezza della traversa ad arco fosse necessaria a consentire questo movimento senza impacci, cioè senza che i bracci si urtassero nello scattare in avanti o ostacolassero, frapponendosi, la corsa del proietto. ${ }^{18}$ In base a questi dati, ci si è spinti anche oltre e si è proposto che tutte le cheiroballistrae avessero bracci correnti verso l'interno (ipotesi nota come "Inswinging Theory"). ${ }^{19} \mathrm{Chi}$ scrive ritiene che sia saggio, al momento, non prendere una posizione netta: tuttavia vuol provarsi egualmente nel cercare di sistemare i dati in un quadro generale coerente.

L'invenzione della cheiroballistra provvide l'esercito romano della migliore lanciagiavellotti che si fosse mai vista. Tuttavia, è lecito supporre che i lanciapietre rimasero, per qualche tempo, il vecchio modello a griglia palintona lignea. ${ }^{20}$ Diversi architecti legionarii devono

\footnotetext{
${ }_{15}$ Per altri reperti D. Baatz, Bauten und Katapulte des römischen Heeres (Stüttgart 1994), $127-135$.

${ }^{16}$ Vegezio, Epitoma rei militaris 2.15; 3.14 (manuballistarii); 4.22 (l'antico nome della manuballista era scorpio).

${ }^{17} \mathrm{La}$ tragula era un corto giavellotto a punta quadrata dotato di propulsore. Cfr. M.C. Bishop and J.C.N. Coulston, Roman Military Equipment (London 1993), 160-162; M. Feugère, Weapons of the Romans (Charleston 2004), 183-185.

${ }_{18}^{18}$ Iriarte 2000, op. cit. (n. 3), 61.

19 A. Iriarte, 'The Inswinging Theory', Gladius 23 (2003), 111-140.

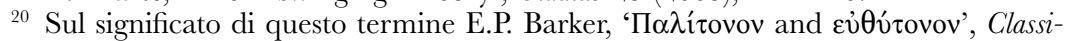
cal Fournal 14 (1920), 82-86; Marsden 1969, op. cit. (n. 1), 23; Marsden 1971, op. cit.
} 
aver provato a costruire un lanciapietre a griglia metallica: un passo ovvio e consequenziale quando si considerino le superiori prestazioni delle cheiroballistrae lanciagiavellotti rispetto alle loro precedenti di legno. Scagliare pietre però comporta problemi diversi dallo scagliare giavellotti. Le formule di calibrazione usate dagli antichi si basavano, ad esempio, su due parametri diversi: la lunghezza del dardo per le lanciagiavellotti e il peso del proiettile per i lanciapietre, ${ }^{21}$ e i diversi problemi da affrontare e risolvere delle due tipologie di macchine da lancio antiche sono all'origine del loro differenziarsi in lanciagiavellotti eutitone e lanciapietre palintoni. I vari tentativi degli ingegneri romani di costruire una cheiroballistra lanciapietre utilizzando lo stesso schema della sua versione lanciagiavellotti devono essere risultati insoddisfacenti: nell'ambito di questi tentativi, qualcuno avrà avuto l'idea di ottenere una spinta più accentuata per il pesante proietto litico consentendo una maggiore corsa angolare dei bracci (mentre per le lanciagiavellotti si continuò ad usare l'impianto tradizionale con i bracci all'esterno). Per ottenere la massima prestazione possibile si sarà pensato di realizzare un pezzo con i bracci correnti all'interno. L'innovazione avrà avuto una certa diffusione ed uno dei lanciapietre così costruiti è giunto fino a noi con i reperti di Orşova. In ogni caso, per un motivo o per l'altro, anche le macchine così costruite devono essere state giudicate insufficienti o non rispondenti ai bisogni dal momento che il progetto fu abbandonato, fra terzo e quarto secolo d.C., in favore di un altro: quello dell'onager, l'unico modello di lanciapietre che Ammiano Marcellino e Vegezio mostrano di conoscere. Va sottolineato che questa ricostruzione, pur se tiene conto, conciliandole, di tutte le teorie avanzate fin'ora e basata su tutta la documentazione disponibile, è largamente ipotetica perché l'evidenza in nostro possesso è ben lungi dall'essere completa. Non si può escludere che nuovi ritrovamenti possano completamente sconvolgere il quadro delineato sopra. ${ }^{22}$

(n. 2), 44-45; Y. Garlan, Recherches de poliorcétique grecque (Athènes et Paris 1974), 223 n. 2. Cfr. anche P. Fleury, 'Vitruve et la nomenclature des machines de jet romaines', Revue des Études Latines 59 (1981), 216-234; P. Fleury, 'Le vocabulaire latin de la mécanique' in P. Radici Colace (ed.), Atti del secondo Seminario Internazionale di Studi sui Lessici Tecnici Greci e Latini. Messina, 14-16 dicembre 1995 (Napoli 1997), 27-40.

${ }^{21}$ Sulle formule di calibrazione e le problematiche connesse Marsden 1969, op. cit. (n. 1), 24-47.

${ }^{22}$ Vorrei qui richiamare l'attenzione su un passo di Vitruvio (10.11.1) quasi ignorato da tutti gli esegeti: "Ballistarum autem rationes variae sunt et differentes, unius effectus comparatae. Aliae enim vectibus, suculis, nonnullae polyspastis, aliae ergatis, quaedam etiam tympanorum torquentur rationibus". Egli dice espressamente che esistono molti tipi di ballistae, cioè lanciapietre, 
L'onager è l'unico pezzo d'artiglieria di cui si possa dire con sicurezza che si distaccava completamente dallo schema costruttivo fusto-slitta tipico delle artiglierie a torsione greco-romane. ${ }^{23}$ Esso aveva i pregi di unire un'estrema semplicità d'impianto ad una grande potenza ed una notevole gittata. Nel quarto secolo d.C. costituiva il lanciapietre tipico usato dai Romani, ma il progetto è probabilmente più antico: ${ }^{24}$ Filone di Bisanzio (200 a.C. circa) tramanda, in una lista di macchine difensive per far precipitare massi contro i lavori d'assalto degli assedianti alla base delle mura, un pezzo chiamato $\mu$ ovó $\gamma \kappa \omega v$ (letteralmente "monobraccio"). ${ }^{25}$ Dopo questo accenno, il $\mu$ ovó $\gamma \kappa \omega v$ sembra sparire per trecento anni dalle fonti, fino a riapparire all'improvviso negli scritti di Apollodoro di Damasco come termine di paragone per un particolare dispositivo bellico da lui descritto. ${ }^{26}$ L'onager appare in pieno quarto secolo d.C. nelle pagine di Ammiano Marcellino, ${ }^{27}$ il quale lo descrive un po' più in dettaglio e lo presenta come ben diffuso ed utilizzato

e che egli ne descrive solo uno (oggi considerato invece - e a torto - come l'unico in servizio nell'esercito romano), quello più razionale perché meglio rispondente ai criteri geometrici delle formule di calibrazione. Ad uno dei pezzi accennati da Vitruvio potrebbe alludere anche Erone, Belopoeica 84.9-85.6.

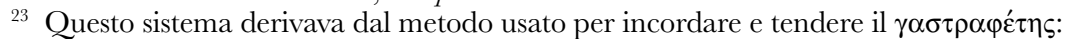
Marsden 1969, op. cit. (n. 1), 5-12. Per i dettagli: Erone, Belopoeica 76.6-79.5; Vitruvio 10.10.3. Cfr. Marsden 1971, op. cit. (n. 2), 194-195; Callebat-Fleury 1986, op. cit. (n. 2), 207-210; Wilkins 2000, op. cit. (n. 2), 81-82; Russo 2002, op. cit. (n. 2), 130-137.

${ }^{24}$ Secondo Barker 1920, op. cit. (n. 20), 85 la catapulta eutitona inventata, secondo Diodoro Siculo (14.41) a Siracusa nel 399 a.C., sarebbe stata un incrocio tra il

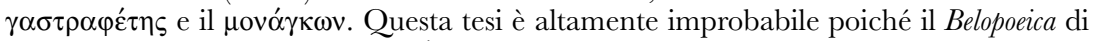
Erone, sunto dai perduti 'Y $Y$ o $\mu v \eta$ $\mu \alpha \tau \alpha$ di Ctesibio di Alessandria del III secolo a.C., si struttura come una storia delle macchine da lancio da quelle a flessione d'un arco composito a quelle a torsione di fasci di tendini, e non accenna al $\mu$ ovó $\gamma \kappa \omega v$. Cfr. Marsden 1969, op. cit. (n. 1), 3. G. Rawlinson, Five Great Monarchies of the Eastern World, I (London 1871), 472 riferisce di aver visto in un bassorilievo assiro la rappresentazione

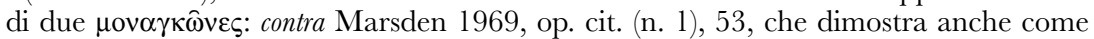
i presunti pezzi d'artiglieria nominati nella Bibbia (Cronache 2.26.15, Ezechiele 4.2 e 21.22) non siano tali. I. Pimouguet Pedarros, 'L'apparition des premiers engins balistiques dans le monde grec et hellénisé: un état de la question', Revue d'Études Anciennes 102 (2000), 5-26 esclude che i lanciapietre possano essere stati inventati dai Persiani nel VI-V secolo a.C. Cfr. P. Briant, 'A propos du boulet de Phocée', Revue des Études Anciennes 96 (1994), 111-114.

${ }^{25}$ Filone di Bisanzio, Mechanica 5.3.10. Garlan 1974, op. cit. (n. 20), 377-378. Non può essere escluso però che questo accenno sia una inserzione bizantina.

${ }^{26}$ Apollodoro di Damasco, Poliorcetica 188.2-9; O. Lendle, Schildkröten: Antike Kriegsmaschinen in Poliorketischen Texten (Wiesbaden 1975), 93-96; O. Lendle, Texte und Untersuchungen zum technischen Bereich der antiken Poliorketik (Wiesbaden 1983), 26-28; D. Sullivan (ed.), Siegecraft: two tenth-century instructional manuals by "Heron of Byzantium" (Washington 2000), 221.

27 Ammiano 23.4.4-7. 
nell'esercito tardoromano. ${ }^{28}$ La descrizione fornitaci da Ammiano sembra riflettere le varie fasi dell'assemblaggio. Non è il caso di esaminarne dettagliatamente l'impianto: basterà notare che dal punto di vista tecnico la macchina era l'equivalente della sola semigriglia di un pezzo a torsione posizionata orizzontalmente al piano d'appoggio invece che verticalmente $\mathrm{e}$, nonostante le difficoltà di brandeggio testimoniate dallo stesso Ammiano, ${ }^{29}$ la semplicità del progetto, la facilità di montaggio $\mathrm{e}$ la terrificante potenza ottenuta anche grazie alle leggi di conservazione del momento angolare sfruttate dalla rotazione della fionda, applicata alla sommità del braccio per allocare il proietto in fase di lancio (la quale imprimeva un surplus di accelerazione e potenza) ne facevano un'arma efficacissima e funzionale..$^{30}$

Quando e perché l'onager divenne il lanciapietre standard dell'esercito romano? A questa domanda è impossibile dare una risposta precisa. Tra le rovine del forte di High Rochester in Inghilterra sono venute alla luce due epigrafi che ricordano la costruzione di una piattaforma per artiglieria (ballistarium) durante il regno di Elagabalo e la ricostruzione o il restauro della stessa sotto Alessandro Severo. ${ }^{31}$ Il sito ospitava, nell'epoca in questione, la Cohors I fida Vardullorum equitata milliaria, ${ }^{32}$ un'unità di ausiliari, il che pone infiniti problemi sul rapporto che intercorreva fra auxilia e artiglierie, poiché queste ultime erano un'esclusiva legionaria. ${ }^{33} \mathrm{E}$ stato ipotizzato che il ballistarium in questione potesse identificarsi con la postazione che, secondo Ammiano, ${ }^{34}$ doveva essere eretta per posizionare l'onager in batteria e che quindi questo pezzo fosse già di normale utilizzo nel primo quarto del terzo secolo d.C. ${ }^{35}$ L'ipotesi non è da scartare a priori, ma non può essere argomentata.

\footnotetext{
${ }^{28}$ Ad esempio Ammiano 19.2.7; 20.7.10; 24.2.13; 24.4.28; 31.15.12. P.E. Chevedden, 'Artillery in Late Antiquity: prelude to the Middle Ages' in I. Corfis and M. Wolfe (eds.), The Medieval City under Siege (Woodbridge 1995), 131-173; D. Baatz, 'Katapulte und mechanische Handwaffen des spätrömischen Heeres', Fournal of Roman Military Equipment Studies 10 (1999), 5-19.

${ }_{29}$ Ammiano 19.7.6.

${ }^{30}$ V.G. Hart and M.J.T. Lewis, 'Mechanics of the onager', Journal of Engineering Mathematics 20 (1986), 345-365.

${ }^{31}$ E. Birley, Research on Hadrian's Wall (London 1961), 242-244; D.B. Campbell, 'Ballistaria in first to mid-third century Britain: a reappraisal', Britannia 15 (1984), 75-84;CIL 5.1044-1045; CIL 5.1046.

${ }^{32}$ Campbell 1984, op. cit. (n. 31), 80-82.

${ }_{33}$ Marsden 1969, op. cit. (n. 1), 184.

${ }_{34}$ Ammiano 23.4.5.

35 Una ipotasi di I.A. Richmond, 'The Romans in Redesdale', in M.H. Dodds (ed.), History of Northumberland.15 (Newcastle 1940), 97; Marsden 1969, op. cit. (n. 1), 191.
} 
La piattaforma poteva essere stata eretta per ospitare anche altri pezzi d'artiglieria, ${ }^{36}$ niente dice che solo l'onager avesse bisogno di questo tipo di opera accessoria per stare in batteria. ${ }^{37}$

Nel 279 d.C. il governatore della Licia-Panfilia, Terenzio Marciano, assediò la roccaforte di un brigante locale, ${ }^{38}$ Cremna in Pisidia, ${ }^{39}$ costringendola alla resa. ${ }^{40}$ I lavori d'assedio dei Romani, riemersi dagli scavi condotti sul sito, ${ }^{41}$ comprendevano una gigantesca collinetta artificiale eretta di fronte alla principale posizione difensiva di Cremna. ${ }^{42}$ La collinetta era chiaramente destinata ad ospitare pezzi d'artiglieria. All'interno delle fortificazioni di Cremna sono emerse molte palle per catapulta del peso di circa $25 \mathrm{Kg} .{ }^{43} \mathrm{Il}$ peso è indicativo, poiché questo è proprio il calibro del più grosso pezzo di artiglieria vitruviano, la ballista palintona da un talento. ${ }^{44}$ Niente vieta di pensare che i proietti in questione potessero essere stati scagliati da un onager, ma secondo tutta la tecnica costruttiva di macchine da lancio della tradizione ellenistica e vitruviana ogni parte della macchina, anche la più piccola, rispondeva ad un canone rigidamente determinato che metteva in relazione tra loro il peso del proietto da scagliare e i diametri dei fasci da porre in torsione. ${ }^{45}$ Naturalmente, non era necessario fare ogni volta i calcoli: ben presto le misure si standardizzarono ed è facile imaginare che gli artiglieri romani venissero istruiti a costruire un determinato numero di tipi di pezzi di calibro ben preciso (da cinque mine, da dieci mine, da un talento...) e, forse, venissero ad essi fornite anche tabelle con misure predeterminate, come agli artiglieri della Prima Guerra Mondiale venivano date tavole logaritmiche che correlavano l'alzo alla gittata dei cannoni, senza che essi dovessero procedere ogni volta a calcolare

\footnotetext{
${ }^{36}$ Campbell 1986, op. cit. (n. 9), 122.

${ }^{37}$ Campbell 1984, op. cit. (n. 31), 82-84.

${ }^{38}$ S. Mitchell, 'Native rebellion in the Pisidian Taurus' in K. Hopwood (ed.), Organised Crime in Antiquity (London 1999), 155-175.

39 S. Mitchell, Cremna in Pisidia, an Ancient City in Peace and in War (London 1995).

${ }^{40}$ Zosimo $1.69-70$.

${ }^{41}$ S. Mitchell, 'The siege of Cremna' in D.H. French and C.S. Lightfoot (eds.), The Eastern Frontier of the Roman Empire 1 (Ankara 1988), 311-328.

42 S. Mitchell, 'Archaeology in Asia Minor 1985-1989', Archaeological Reports 36 (1989-1990), 83-131.

${ }_{43}$ Mitchell 1989-1990, op. cit. (n. 42), 123-124.

44 Vitruvio 10.11.3.

45 A.G. Drachmann, 'Remarks on the ancient catapults' in Actes du septième congrès international d'histoire des sciences (Jerusalem 1953), 280-282; Marsden 1969, op. cit. (n. 1), 24-45; Marsden 1971, op. cit. (n. 2), 197-200; Russo 2002, op. cit. (n. 2), 189-198.
} 
questa relazione..$^{46}$ È forte il sospetto, data la scarsa o nulla variabilità del peso dei proietti trovati a Cremna, che essi siano stati scagliati da un lanciapietre palintono del tipo descritto da Vitruvio e che questa macchina fosse dunque ancora in servizio alla fine del terzo secolo d.C. ${ }^{47}$ Certo, è possibile che i proietti di Cremna siano stati scagliati da onagri. Per essere adottato nella maniera massiccia testimoniata da Ammiano, questo pezzo doveva assolvere tutti i compiti dei suoi predecessori e anche di più: ${ }^{48}$ poteva dunque ben lanciare palle da un talento. Ma, come testimoniano le ricostruzioni moderne ${ }^{49}$ l'onager era un pezzo più flessibile di una ballista vitruviana, soggetta a rompersi se il proietto era troppo leggero o ad imprimere al proietto medesimo un'enegia cinetica insufficiente se questo era troppo pesante. Gli assedianti di Cremna non avrebbero avuto ragione di penare per ridurre pietre in calibri così omogenei e uniformi se fossero stati equipaggiati con onagri. ${ }^{50}$

A mio parere, l'adozione dell'onager come modello dei lanciapietre standard in seno all'esercito romano è da riconnettere in qualche modo con i dominati di Diocleziano e Costantino. Particolarmente il primo, con la sua politica di fortificazione dell'impero, è indiziato. ${ }^{51}$ L'onager, lo si è detto sopra, esisteva ben prima dei tempi di Ammiano. Gli accenni ad esso ricorrono sempre in un contesto ossidionale. Era una macchina strettamente connessa con la difesa di una cortina muraria. Un elemento

${ }^{46}$ Le misure date da Vitruvio 10.11.3-9 presuppongono chiaramente un prontuario di questo tipo.

${ }^{47}$ Per un'analisi sull'uniformità delle classi di peso, e dei relativi calibri, dei proiettili ritrovati a Cartagine, Rodi, Pergamo e Marsiglia (tutti per lanciapietre palintoni) Marsden 1969, op. cit. (n. 1), 79-83. Cfr. Russo 2002, op. cit. (n. 2), 180-186 per quelli ritrovati a Pompei, risalenti a Silla. Centinaia di proiettili ritrovati ad Hatra non sono stati, purtroppo, né catalogati né studiati: D.B. Campbell, Greek and Roman Artillery 399 $B C-A D 363$ (London 2003), 20.

${ }^{48}$ Cfr. P.F. Drucker, 'Modern technology and ancient jobs', Technology and Culture 4 (1963), 277-281; O. Pi Sunyer and T. De Gregori, 'Cultural resistance to technological change', Technology and Culture 5 (1964), 247-253.

${ }^{49}$ R. Payne Gallwey, The Crossbow (London 1903), 279-299; E. Schramm, 'Movó $\gamma \kappa \omega v$ und onager', Nachrichten von der Gesellschaft der Wissenschaften zu Göttingen 2 (1918), 259-271; Marsden 1971, op. cit. (n. 2), 254-265.

${ }^{50} \mathrm{Si}$ pensi, ad esempio, alla disomogeneità di forma e peso dei proiettili di mangano ritrovati nel castello medioevale di Saranda Kolones a Cipro: H.S. Megaw, 'Supplementary excavations on a castle site at Paphos, Cyprus, 1970-1971', Dumbarton Oaks Papers 26 (1972), 322-343; J. Rosser, 'Excavations at Saranda Kolones, Paphos, Cyprus 1981-1983', Dumbarton Oaks Papers 39 (1985), 81-97.

${ }^{51}$ Sulle fortificazioni tardoromane: S. Johnson, Late Roman Fortifications (London 1983); A. Johnson, Roman Forts (London 1983); J. Lander, Roman Stone Fortifications (Oxford 1984); P. Southern and K.R. Dixon, The Late Roman Army (London-New York 1996), 127-167; H. Elton, Warfare in Roman Europe A.D. 350-425 (Oxford 1996), 155-174. 
sopra tutti lo testimonia: il fatto che l'onager fosse brandeggiabile solo con molta difficoltà. ${ }^{52}$ I difensori, potendosi avvalere delle postazioni di tiro preparate sulle mura durante i periodi di pace, ${ }^{53}$ non avevano a soffrire troppo della poca brandeggiabilità dell'onager, che veniva posto in batteria già in un punto con il campo di tiro ideale. Inoltre, la flessibilità di questo pezzo nello scagliare pietre di vario peso metteva al riparo dalla scarsità di munizioni adatte e consentiva l'uso anche di munizioni di fortuna (tegole, mattoni) in caso di necessità. L'erezione dei numerose fortificazioni durante il regno di Diocleziano e Costantino può aver dato impulso allo sviluppo di questa macchina il cui progetto giacque per secoli semi-dimenticato nei manuali di $\pi \alpha \rho \alpha \sigma \kappa \varepsilon v \alpha \sigma \tau \imath \kappa \grave{\alpha}$ (le preparazioni per resistere ad un assedio).

L'adozione dell'onager rappresentò un considerevole progresso nel campo delle artiglierie meccaniche. Esso offriva svariati vantaggi che non devono essere sottovalutati: era più semplice, facile e veloce da assemblare rispetto ad una ballista vitruviana; questa era composta da numerosi componenti che non figurano nel progetto dell'onager e che richiedevano molta attenzione nella realizzazione e nel montaggio, e non poca abilità carpentieristica e preparazione matematica da parte degli artiglieri. ${ }^{54}$ Anche l'unico punto in cui l'onager poteva essere inferiore ad una ballista vitruviana, la brandeggiabilità, è probabile che debba essere riconsiderato: Vegezio accenna ad un affusto mobile trainato da buoi. ${ }^{55}$

Quello dell'adozione dell'onager fu un progresso innescato dalla crisi del terzo secolo d.C., che portò a rivalutare un vecchio modello di macchina usato nella difesa muraria, facendone scoprire i pregi. Fu la crisi del terzo secolo d.C. la molla che portò alla soluzione del problema postosi, per l'esercito romano, nel secondo secolo d.C. dopo l'introduzione della cheiroballistra, cioè quello di dotarsi di un lanciapietre più semplice e potente, come più semplice e potente era la nuova

${ }^{52}$ Ammiano 19.7.6-7.

${ }^{53}$ S. Johnson 1983, op. cit. (n. 49), 31-54 e 78-81; A. Johnson 1983, op. cit. (n. 49), 53-55; Lander 1984, op. cit. (n. 49), 198-262 e 302-306.

${ }_{54}$ Marsden 1971, op. cit. (n. 2), 263-265.

55 Vegezio, Epitoma rei militaris 2.25: "Onagri... in carpentis bubus portantur armatis". Questa frase potrebbe apparire decisiva, ma bisogna considerare che Vegezio può aver scritto riferendosi alla antiqua legio (che può aver conosciuto lanciapietre palintoni montati su carri) usando il vocabolo corrente ai suoi giorni per designare il lanciapietre. 
lanciagiavellotti entrata in linea con Traiano. ${ }^{56}$ Un progresso talmente funzionale, talmente rispondente alle esigenze della classe dominante che aveva prodotto questo bisogno da sopravvivere alla scomparsa e/o trasformazione di questa stessa classe dominante poiché l'onager, ovvero i suoi diretti discendenti, il mangano, ${ }^{57}$ la petriera e il trabucco, ${ }^{58}$ restarono in uso fino alla metà del XVI secolo. ${ }^{59}$

Un fenomeno analogo e comparabile è l'adozione delle batterie di lanciarazzi in seno agli eserciti contemporanei. I primi razzi, come è noto, furono usati in Cina dal settimo secolo d.C., forse addirittura dal primo secolo d.C. ${ }^{60}$ In Europa essi furono, curiosamente, contemporanei degli ultimi trabucchi e restarono sporadicamente in uso fino alla Guerra Civile Americana ${ }^{61}$ I progressi nella costruzione dei cannoni sfavorirono l'ulteriore sviluppo di questo tipo di arma che venne dimenticata restando solo per le segnalazioni luminose. Nella seconda guerra mondiale l'esercito russo, a corto di materiale bellico e bisognoso di un'arma che offrisse grande potenza e concentrazione di fuoco, reintrodusse i razzi, montandoli in batteria sul cassone di banalissimi camion. ${ }^{62}$ La "Katiusha" si rivelò un'arma così micidiale, semplice, funzionale e

${ }^{56}$ Cfr. Le considerazioni generali di W. Kaempffert, 'War and Technology', The American Fournal of Sociology 46 (1941), 431-444; R.P. Multhauf, 'The scientist and the improver of technology', Technology and Culture 1 (1959), 38-47; B.C. Hacker, 'Greek catapults and catapult technology: Science, technology and war in the ancient world' Technology and Culture 9 (1968), 34-50; R.F. Weigley, 'War and the paradox of technology', International Security 14 (1989), 192-202; G. Raudzens, 'War-winning weapons: the measurement of technological determinism in military history', The fournal of Military History 54 (1990), 403-434; A. Roland, 'Theories and models of technological change: semantics and substance', Science, Technology and Human Values 17 (1992), 79-100; A. Roland, 'Science, technology and war', Technology and Culture 36 (1995), 83-100.

${ }^{57}$ B.S. Bachrach, 'Medieval siege warfare', The Journal of Military History 58 (1994), $119-133$.

${ }^{58}$ La petriera: W.T.S. Tarver, 'The traction trebuchet: a reconstruction of an early medieval siege engine', Technology and Culture 36 (1995), 136-167; il trabucco: P.E. Chevedden, 'The invention of the counterweight trebuchet: a study in cultural diffusion', Dumbarton Oaks Papers 54 (2000), 71-116.

${ }_{59}$ L'ultimo, inglorioso, uso del trabucco fu a Tenochtitlàn nel 1521: Bernal Diaz del Castillo, Historia de la conquista de Mexico 155; Bernardino de Sahagùn, Historia de la Nueva España 12.37.

${ }^{60} \mathrm{~W}$. Ling, 'On the invention and use of gunpowder and firearms in China', Isis 37 (1947), 160-178.

${ }^{61}$ E.M. Emme, 'Introduction to the history of rocket technology', Technology and Culture 4 (1963), 377-383; E.M. Emme, 'International history of rocketry and astronautics symposium: Constance, W. Germany, october 1970', Technology and Culture 12 (1971), 477-486; P.D. Olejar, 'Rockets in early american wars', Military Affairs 10 (1946), 16-34; R.W. Donnelly, 'Rocket batteries of the Civil War', Military Affairs 25 (1961), 69-93.

${ }_{62}$ G.A. Tokaty, 'Soviet rocket technology', Technology and Culture 4 (1963), 515-528. 
devastante, pur nella sua rozzezza, che ad essa possono essere ascritte molte vittorie tattiche dell'Armata Rossa, non ultima Stalingrado, dove le batterie di "Katiusha" appostate sulla sponda orientale del Volga annientavano sistematicamente ogni conquista tedesca. Oggi il razzo ha soppiantato quasi del tutto il cannone. Un'arma antica, fino ad allora poco usata, fu ripescata sotto la spinta della necessità per essere utilizzata in un contesto nuovo, dove si rivelò un progresso tremendamente efficace e funzionale, al punto da sostituire quasi completamente la sua concorrente. ${ }^{63}$

Napoli, settembre 2006

63 L. White jr., 'The act of the invention: causes, contexts, continuities and consequences', Technology and Culture 3 (1962), 486-500. 